\author{
Ecología
}

\title{
Escarabajos fitófagos (Coleoptera: Scarabaeidae) en un fragmento de bosque seco tropical del departamento del Atlántico, Colombia
}

\author{
Phytophagous scarab beetles (Coleoptera: Scarabaeidae) in a fragment of tropical dry forest, \\ Atlántico Department, Colombia \\ Sandy García-Atencia ${ }^{\mathrm{a}, *}$, Neis Martínez-Hernández ${ }^{\mathrm{a}}$ y Luis Carlos Pardo-Locarno ${ }^{\mathrm{b}}$ \\ ${ }^{a}$ Grupo de Investigación Biodiversidad del Caribe Colombiano, Facultad de Ciencias Básicas, Universidad del Atlántico. Ciudadela Universitaria Km 7 vía \\ Puerto Colombia, Apartado 1890 Barranquilla, Colombia

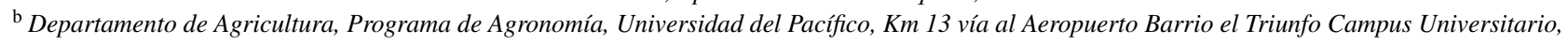 \\ Buenaventura, Colombia
}

Recibido el 12 de enero de 2015; aceptado el 12 de mayo de 2015

Disponible en Internet el 31 de agosto de 2015

\begin{abstract}
Resumen
Los ambientes forestales de zonas de vida secas y muy secas del Caribe colombiano han sido profundamente impactados y a la fecha quedan muy pocos remanentes y rastros de su dinámica biológica original; por ello, en esta investigación se planteó estudiar la estructura y la variación estacional de los escarabajos fitófagos (Coleoptera: Scarabaeidae) en la Reserva Campesina La Montaña. Se realizaron 9 muestreos, desde enero hasta septiembre de 2011, y se instalaron 4 trampas de luz (2 BL y 2 WL) entre las 18:00 y las 24:00 h. Se capturaron 3,007 individuos y 18 especies agrupados en 3 subfamilias: Rutelinae (7 especies), Melolonthinae (6 especies) y Dynastinae (5 especies); sobresalió Melolonthinae con la mayor abundancia (2,907 individuos), y Liogenys quadridens fue la especie más abundante (1,667 individuos), reconocida por ser una plaga de cultivos semestrales, de amplia distribución en el Caribe colombiano. La mayor riqueza (16 especies) se observó en mayo y la mayor abundancia $(1,421)$, en abril, lo que coincidió con la época de las primeras lluvias en la región, lo que pone de manifiesto una marcada estacionalidad que quedó demostrada con el análisis de similitud Anosim $(R=0.277, p<0.003)$.

Derechos Reservados (C) 2015 Universidad Nacional Autónoma de México, Instituto de Biología. Este es un artículo de acceso abierto distribuido bajo los términos de la Licencia Creative Commons CC BY-NC-ND 4.0.
\end{abstract}

Palabras clave: Scarabaeidae; Riqueza; Abundancia; Estacionalidad; Caribe seco

\begin{abstract} Anosim $(R=0.277, p<0.003)$. Creative Commons CC License BY-NC-ND 4.0.

Keywords: Scarabaeidae; Richness; Abundance; Seasonality; Dry Caribbean

\footnotetext{
* Autor para correspondencia.

Correo electrónico: sandyga.01@ gmail.com (S. García-Atencia).

La revisión por pares es responsabilidad de la Universidad Nacional Autónoma de México.
}

Dry forests areas in the Caribbean region of Colombia have been deeply impacted lagging far precious few remnants and traces of its original dynamics, so the objective of this research is to study the structure and seasonality variation of phytophagous scarab beetles (Coleoptera: Scarabaeidae) in the Reserva Campesina La Montaña. Nine samples were obtained from January to September 2011. Four points distanced approximately $150 \mathrm{~m}$ were marked. In each one, a light trap was settled, for a total of 4 ( 2 white and 2 violet) operating since 18:00 until 24:00 h. 3,007 individuals and 18 species clustered in 3 subfamilies were collected: Rutelinae (7 species), Melolonthinae (6 species) and Dynastinae (5 species). Melolonthidae were the most abundant and Liogenys quadridens its most abundant specie (1,667 individuals), characterized by an important pest of semestral crops, and wide distribution in the Colombian Caribbean. The highest richness (16) was obtained in May, while April presented higher abundance $(1,421)$, which coincides with the beginning of the rainy season, showing a marked seasonality showed by the analysis of similarity

All Rights Reserved (C) 2015 Universidad Nacional Autónoma de México, Instituto de Biología. This is an open access item distributed under the 


\section{Introducción}

En la región Caribe colombiana la extension de bosque seco tropical (BST) está reducida debido a las presiones de agricultura y ganadería que ocasionan, además de fragmentación, la transformación a estados sucesionales intermedios, rodeados por matrices de transformación (Miles et al., 2006; PortilloQuintero y Sánchez-Azofeifa, 2010). En estos fragmentos los insectos constituyen una parte importante de la diversidad biológica, los cuales son considerados indicadores de heterogeneidad de los hábitats, de la diversidad de ecosistemas y de su grado de intervención o de estrés ambiental (Cano y Schuster, 2009). Se ha registrado que los insectos y otros artrópodos de bosque seco se ven muy influenciados por la disponibilidad hídrica durante el año y por cambios fenológicos en la vegetación, lo que incide en la disminución de sus poblaciones en los periodos de bajas o altas precipitaciones (Castellanos, Pardo-Locarno, Carabalí, Doria, 2011; Escobar, 1997; Martínez, Cañas, Rangel, Barraza, Montes y Blanco, 2010; Martínez, García, Sanjuán, Gutiérrez y Contreras 2010). Lo anterior puede incidir en la dinámica ecológica de grupos funcionales como los insectos fitófagos de la familia Scarabaeidae, los cuales son uno de los primeros eslabones en el flujo trófico en ecosistemas como el BST (Pardo-Locarno, 2013; Schëu, 2002). Cualquier alteración ambiental o antrópica que afecte la comunidad local de estos organismos también afectará de manera directa o indirecta los procesos ecológicos como la descomposición de detritus y, en consecuencia, el flujo de nutrientes (Lassau, Hochuli, Cassis y Reid, 2005). Además, por su variedad de gremios tróficos pueden reflejar la dinámica del ecosistema, como es el caso de las especies sapro-xilófagas que cumplen un importante papel en los bosques como degradadores primarios de madera (Morón, 1985, 1994).

En Colombia, los estudios de evaluación de la estacionalidad de esta familia de insectos han estado enfocados al sector agrícola, desarrollados por Londoño (1999), Pardo-Locarno, Montoya y Schoonhoven (2003), Pardo-Locarno, Montoya, Schoonhoven, Morón (2003), Útima y Vallejo (2008), Yepes, Pardo, Pérez y Quiroz (2000), aunque se destaca la lista de especies en ecosistemas pluviales realizado por Neita, Orozco y Ratcliffe (2006) y las fichas bioecológicas y clave para adultos, realizadas por Pardo-Locarno (2013) en BST del plan aluvial del río Cauca. En el Caribe colombiano, se han realizado estudios sobre escarabajos Melolonthidae consistentes en registros de distribución (Gasca-Álvarez y Amat-García, 2010; García-Atencia y Martínez-Hernández, 2015; Pardo-Locarno, González, Pérez, Yepes y Fernández, 2012; Restrepo-Giraldo, Morón, Vallejo, Pardo-Locarno y López-Ávila, 2003; Suárez y Amat, 2007), aunque se presume que faltan especies por incorporar en tales inventarios, debido a la falta de estudios con técnicas más específicas para este grupo o la poca representatividad en las colecciones nacionales.

Teniendo en cuenta la importancia biológica, ecológica y de conservación que implica el estudio de los escarabajos fitófagos y el planteamiento de una variación hipotética del gremio, debido a atributos biofísicos del medio natural, este estudio investiga la estructura y la variación estacional de la riqueza y abundancia de los escarabajos fitófagos (Scarabaeidae "pleurosticti") en un fragmento forestal de la Reserva Campesina la Montaña (RCM) en el departamento del Atlántico; así como también analizar la efectividad del tipo de luz en los muestreos de diversidad de este grupo de escarabajos.

\section{Materiales y métodos}

Con una extensión de 47 ha, la RCM, declarada así desde 1996 (Decreto 1777), se encuentra en el municipio de Juan de Acosta, departamento del Atlántico, Colombia (fig. 1). Se localiza a $10^{\circ} 46^{\prime} 2.6^{\prime} \mathrm{N}, 75^{\circ} 02^{\prime} 34^{\prime \prime} \mathrm{O}$, a una altitud que oscila entre 177 y 250 m, y es parte del distrito Montes de María y Piojó, en la región biogeográfica del Cinturón Árido Pericaribeño (Hernández, 1992). Se trata de un BST con condiciones higrotropofíticas (Holdridge, Grenke, Hatheway, Liang y Tosi, 1971). Su relieve es ondulado, y forma parte de los ecosistemas de serranías, con pendientes entre el 12 y el $25 \%$, con un grado de erosión moderado (Panza et al., 2001). Los meses de mayor precipitación histórica corresponden a agosto y septiembre, con promedios que van desde $100 \mathrm{~mm} / \mathrm{mes}$ hasta $580 \mathrm{~mm} / \mathrm{mes}$, mientras que enero, febrero y marzo corresponden a los meses con menores precipitaciones, que van desde 0 hasta $30 \mathrm{~mm} / \mathrm{mes}$. La temperatura alcanza valores mínimos de $25^{\circ} \mathrm{C}$ y máximos de $33^{\circ} \mathrm{C}$ durante todo el año.

La RCM presenta una vegetación típica conformada por 3 estratos vegetativos de dosel, arbustivo y sotobosque (IAVH, 1998), con grupos representativos como Ceiba Mill, Pseudobombax Dugand, Tabebuia Gomez, Philodendron Schott, Anthurium Schott, Acrocomia Mart, Aristolochia L. y las especies Spondias mombin L., Crescentia cujete L., Pseudobombax septenatum (Jacq.) Dugand, Anacardium excelsum L., Bromelia (L.) Adans., Parinari pachyphylla Rusby, Justia bracteosa (Mildbr.) Leonard, Malvaviscus arboreus Dill. ex Cav, Myrmecodendron costarriscense Britt. y Rose, Petiveria alliiaceae L., Cordia alba (Jacq.) Roem. y Schult, Mangifera indica L., Guazuma ulmifolia Lam., Pereskia quisqueyana (Ekman) Alain, Bursera simaruba (L.) Sarg., Hura crepitans L, y conforma un sistema de selva seca estacional caducifolia rodeada por pequeñas parcelas, donde son comunes las prácticas de ganadería extensiva y agricultura; esta última con cultivos permanentes de maíz, yuca y ají. Además, se encuentra rodeado de fincas ganaderas y se presenta tala y quema de árboles para producción de carbón, extracción de leña y construcción de viviendas, por lo cual su área original se ha reducido durante los últimos años.

Se realizaron muestreos mensuales desde enero hasta septiembre de 2011; en total, 9 muestreos (1 por mes) y 36 muestras, obtenidas así: en el interior del fragmento se marcaron 4 puntos distanciados $150 \mathrm{~m}$, aproximadamente, y se colocó una trampa de luz por punto, en total 4 trampas ( 2 blancas y 2 violetas). Las trampas se instalaron de manera alternada teniendo en cuenta el espectro, desde las 18:00 a 24:00 h.

En cada punto se tomaron las siguientes variables ambientales: humedad relativa (\%), temperatura ambiente (termohigrómetro marca Extech RHT) y del suelo $\left({ }^{\circ} \mathrm{C}\right.$ ) (marca: Thermoworks RT610B-12). Para los valores de precipitación, 


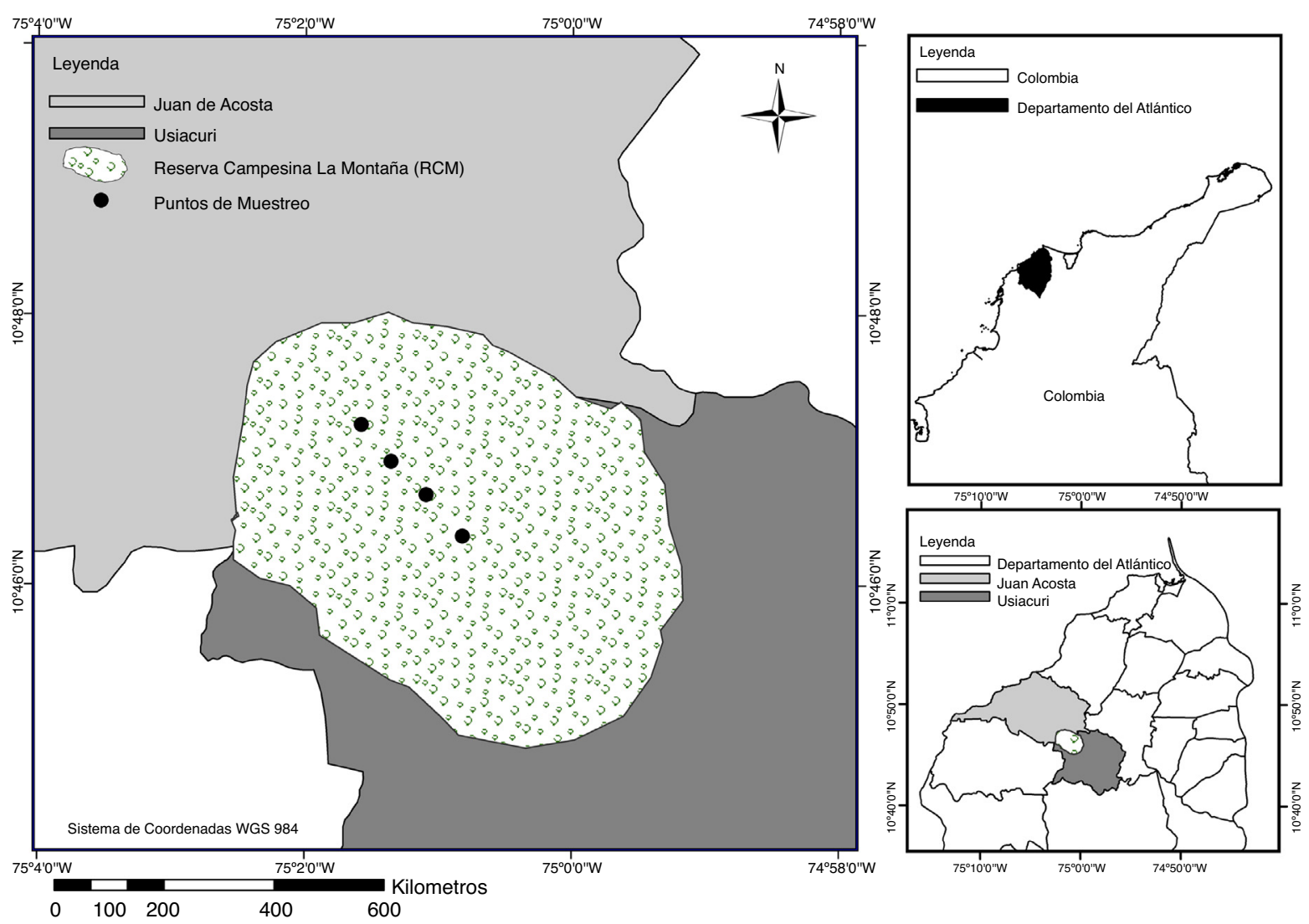

Figura 1. Ubicación de la Reserva Campesina la Montaña, Juan de Acosta, Atlántico, Colombia.

se instaló un pluviómetro marca Hellman en el borde del fragmento, para obtener registros diarios de este parámetro y de esa manera obtener el promedio mensual. Teniendo en cuenta la precipitación, los meses de muestreos se agruparon en 3 épocas: seca, con precipitaciones menores a $15 \mathrm{~mm}$; primeras lluvias, con valores entre $15-150 \mathrm{~mm}$, y lluvias fuertes, con valores por encima de $150 \mathrm{~mm}$.

Las muestras se preservaron en alcohol al $70 \% \mathrm{y}$, posteriormente, se montaron en alfileres entomológicos con su respectivo rótulo. Los individuos se identificaron con las claves propuestas por Delgado, Pérez y Blackaller (2000); Endrödi (1985), Ramírez-Ponce y Morón (2009); SanabriaGarcía, Gasca-Álvarez y Amat-García (2012) y Vallejo y Wolff (2013) y, posteriormente, fueron confirmadas en las colecciones del Museo de Historia Natural-Instituto de Ciencias Naturales (MHN-ICN) y Colección Familia Pardo-Locarno (CFPL-COL). El material producto de este trabajo reposa en la colección de referencia de la Universidad del Atlántico (UARC) y la colección de entomología del Instituto de Ciencias Naturales (ICN), Universidad Nacional de Colombia.

Se determinó la riqueza como el número de especies capturadas por trampa y por muestreo, y la abundancia como el número de individuos. Para evaluar la representatividad de los muestreos, se realizó una curva de acumulación con los estimadores no paramétricos Chao1, ACE y los singletons y doubletons, calculados con el programa EstimateS 9.1 (Colwell, 2013). La diversidad o dominancia se determinó calculando las series de los números de
Hill (N1 y N2) con el programa Primer 6.0 (Clarke y Warwick, 2001). Para determinar si la riqueza, la abundancia y la diversidad presentaron diferencias significativas entre trampas de luz, se utilizó la prueba de Mann-Whitney.

Los escarabajos capturados se agruparon en gremios tróficos (tipo de alimentación de la larva y del adulto) según lo propuesto por Morón y Deloya (1991) y modificados por Deloya, ParraRabla y Delfín-González (2007): rizofilófagos, sapromelífagos, xilofilófagos, saprocaulófagos y xilodepredadores. Con el fin de evaluar si la estructura difiere entre las épocas de seca, primeras lluvias y lluvias fuertes, se realizó una prueba ANOSIM. Para identificar las especies que caracterizaron o tipificaron las épocas de muestreo a través de su abundancia; se utilizó la rutina SIMPER. Para este análisis se tomaron en cuenta las especies que aportaron más del 10\% de la abundancia total. Para estimar en qué medida las variables ambientales como precipitación, humedad relativa ambiental, temperatura ambiental y temperatura del suelo se relacionaron con la variación de la estructura de la comunidad, y se realizó un análisis de correlación canónica, incorporando a aquellas especies que aportaron más a la abundancia durante todos los muestreos.

\section{Resultados}

Se capturaron 3,007 individuos agrupados en 18 especies, 11 tribus y 3 subfamilias (Melolonthinae, Rutelinae y Dynastinae) (tabla 1). La subfamilia más rica (38.9\%) fue Rutelinae, seguida 
Tabla 1

Variación de la riqueza $\left(\mathrm{N}_{0}\right)$, abundancia $(\mathrm{N})$ y diversidad $\left(\mathrm{N}_{1}\right.$ y $\left.\mathrm{N}_{2}\right)$ de escarabajos fitófagos (Scarabaeidae) durante los meses de muestreo en la RCM.

\begin{tabular}{|c|c|c|c|c|c|c|}
\hline Especies & Época seca & Primeras lluvias & Lluvias fuertes & Total & Gremio & Tipo de luz \\
\hline \multicolumn{7}{|l|}{ DYNASTINAE $(\mathrm{S}=5, \mathrm{~N}=18)$} \\
\hline Cyclocephala melanocephala (Fabricius, 1775) & 2 & 0 & 2 & 4 & $\mathrm{Sm}$ & $\mathrm{LB} / \mathrm{LV}$ \\
\hline \multicolumn{7}{|l|}{ Pentodontini } \\
\hline \multicolumn{5}{|l|}{ Oryctini } & $\mathrm{Sc}$ & $\mathrm{LB} / \mathrm{LV}$ \\
\hline $\begin{array}{l}\text { Strategus aloeus (Linnaeus, 1758) } \\
\text { Phileurini }\end{array}$ & \multicolumn{5}{|c|}{ Phileurini } & LB \\
\hline $\begin{array}{l}\text { Phileurus didymus Linnaeus } 1758 \\
\text { MELOLONTHINAE }(\mathrm{S}=10, \mathrm{~N}=2,907) \\
\text { Diplotaxini }\end{array}$ & 0 & 2 & 0 & 2 & $\mathrm{D}$ & LB \\
\hline \multicolumn{7}{|l|}{ Melolonthini } \\
\hline Phyllophaga impressipyga (Frey, 1975) & 0 & 127 & 0 & 127 & $\mathrm{Rf}$ & $\mathrm{LB} / \mathrm{LV}$ \\
\hline P. (P.) menetriesi (Blanchard, 1851) & 0 & 665 & 0 & 665 & $\mathrm{Rf}$ & $\mathrm{LB} / \mathrm{LV}$ \\
\hline $\begin{array}{l}\text { P. pachypyga (Burmeister, 1855) } \\
\text { Sericini }\end{array}$ & \multicolumn{5}{|c|}{ Sericini } & $\mathrm{LB} / \mathrm{LV}$ \\
\hline \multicolumn{7}{|l|}{ Macrodactilini } \\
\hline $\begin{array}{l}\text { Barybas } \text { sp. } \\
\text { RUTELINAE }(\mathrm{S}=7, \mathrm{~N}=82) \\
\text { Anomalini }\end{array}$ & 0 & 37 & 0 & 37 & $\mathrm{Rf}$ & $\mathrm{LB} / \mathrm{LV}$ \\
\hline Paranomala inconstans (Burmeister, 1844) & 0 & 19 & 1 & 20 & $\mathrm{Rf}$ & $\mathrm{LB} / \mathrm{LV}$ \\
\hline Paranomala sp.1 & 0 & 41 & 0 & 41 & $\mathrm{Rf}$ & $\mathrm{LB} / \mathrm{LV}$ \\
\hline Paranomala sp. 2 & 0 & 4 & 1 & 5 & $\mathrm{Rf}$ & $\mathrm{LB} / \mathrm{LV}$ \\
\hline \multicolumn{7}{|l|}{ Geniatini } \\
\hline Leucothyreus sp.1 & 0 & 1 & 0 & 1 & $\mathrm{Xf}$ & LB \\
\hline Leucothyreus sp.2 & 0 & 1 & 0 & 1 & $\mathrm{Xf}$ & LB \\
\hline \multicolumn{7}{|l|}{ Rutelini } \\
\hline Xenopelidnota anomala & 0 & 11 & 2 & 13 & $\mathrm{Xf}$ & $\mathrm{LB} / \mathrm{LV}$ \\
\hline Número de especies $\left(\mathrm{N}_{0}\right)$ & 3 & 17 & 7 & 18 & - & - \\
\hline Número de Individuos & 14 & 2,795 & 198 & 3,007 & - & - \\
\hline Número de Hill $\left(\mathrm{N}_{1}\right)$ & 2.72 & 4 & 1.59 & - & - & - \\
\hline Número de Hill ( $\left.\mathrm{N}_{2}\right)$ & 2.58 & 2.83 & 1.23 & - & - & - \\
\hline
\end{tabular}

D: depredador; LB: luz blanca; LV: luz violeta; Rf: rizofilófago; Sc: saprocaulófago; Sm: sapromelífago; Xf: xilofilófago.

por Melolonthinae con 33.3\%, que, además, presentó la mayor abundancia con el $96.7 \%$ de los individuos; Dynastinae fue la menos diversa y abundante con un 27.8 y un $0.6 \%$, respectivamente. Con respecto a la riqueza por trampa de luz utilizada, 6 de las 18 especies recolectadas fueron exclusivas de la trampa de luz blanca, mientras que las restantes (12), se capturaron con ambos tipos de luz. En cuanto a la abundancia, el mayor valor con 2,277 individuos se presentó en la luz blanca, y en la luz violeta se capturaron 730 individuos. Sin embargo, no se encontraron diferencias significativas en cuanto a la riqueza $(U=146.5, p=0.62)$ y abundancia $(U=121.5, p=0.19)$ de especies por espectro de luz utilizado.

Según los estimadores no paramétricos ACE (19 especies) y Chao1 (21 especies), se capturó entre el 87 y el 99\% de las especies de Scarabaeidae fitófagos esperados para la localidad durante el periodo de muestreo y con el esfuerzo utilizado (fig. 2A). Además de esto, el número de las especies Singletons (Paranomala sp.3, Leucothyreus sp.1 y Leucothyreus sp.2) y Doubletons (Cyclocephala ovulum, Strategus aloeus y Phileurus didymus) se mantienen poco variables a través del tiempo. Las curvas de acumulación de especies observadas por espectro de luz utilizado (fig. 2B) demostraron que la trampa de luz blanca se comportó como una buena técnica de atracción para escarabajos fitófagos de la familia Scarabaeidae "pleurosticti" en el fragmento de BST en el departamento del Atlántico.

La mayor riqueza y abundancia se presentaron en abril (12 especies y 1,421 individuos) y mayo (15 especies y 1,171 individuos), debido a que la mayoría de las especies recolectadas aparecieron solo en este intervalo de tiempo. Estos valores coinciden con la finalización de las primeras lluvias ( $>15 \mathrm{~mm}$ y $<150 \mathrm{~mm}$ ), respectivamente. En estos muestreos, la temperatura ambiente se mantuvo en valores similares (entre 28.7 y $29.4{ }^{\circ} \mathrm{C}$ ), y la temperatura del suelo, entre los 24.7 y $22.7^{\circ} \mathrm{C}$, respectivamente; mientras que la humedad presentó los valores más altos durante esta época (79.6 y 84.5\%) (tabla 2). Por otra parte, la menor abundancia se registró en enero, con la ausencia total de individuos para esta familia (tabla 1). Este mes coincidió con la época seca en la RCM (precipitación $=11.5 \mathrm{~mm}$ ), con valores bajos de humedad relativa $(64.3 \%)$.

Los mayores valores de diversidad de $\mathrm{N}_{1}$ (4) y $\mathrm{N}_{2}$ (2.83) se presentaron en la época de primeras lluvias (tabla 1), coincidiendo con la mayor riqueza y altos valores de abundancia, lo que 

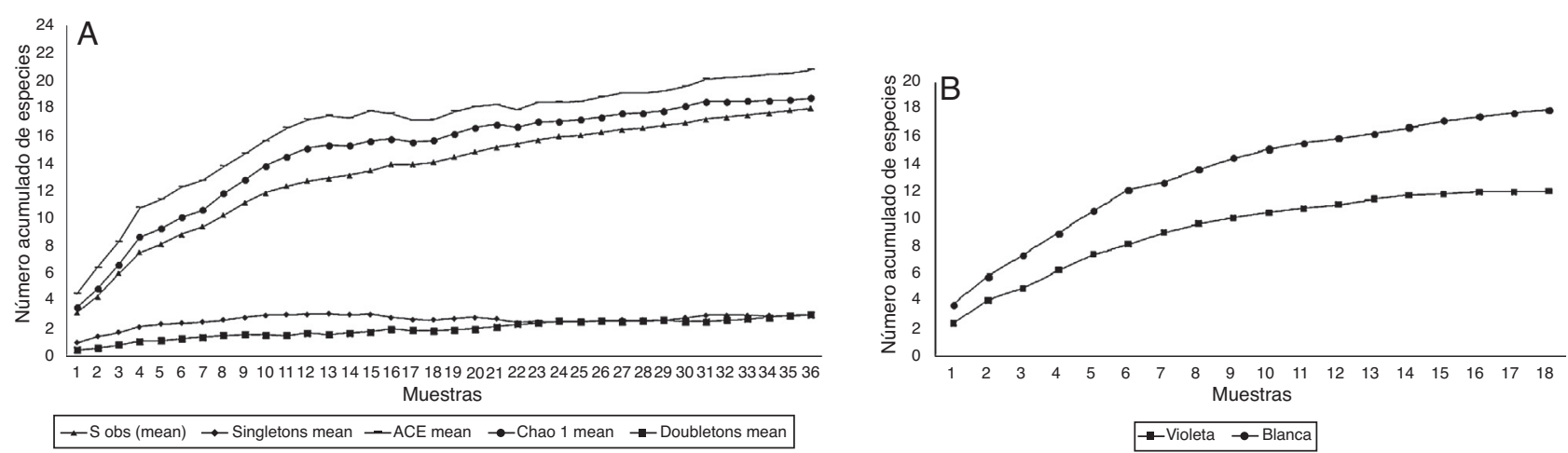

Figura 2. A, curvas de acumulación de especies y B, observadas por espectro en la Reserva Campesina la Montaña, Atlántico, Colombia.

Tabla 2

Variación mensual de las variables ambientales durante los muestreos en la RCM.

\begin{tabular}{|c|c|c|c|c|c|c|c|c|c|}
\hline \multirow[t]{2}{*}{ Variables } & \multicolumn{2}{|c|}{ Época seca } & \multicolumn{3}{|c|}{ Primeras lluvias } & \multicolumn{4}{|c|}{ Lluvias fuertes } \\
\hline & Enero & Febrero & Marzo & Abril & Mayo & Junio & Julio & Agosto & Septiembre \\
\hline Temperatura ambiental $\left({ }^{\circ} \mathrm{C}\right)$ & 28.6 & 26.5 & 32 & 28.7 & 29.4 & 28.9 & 27.1 & 27.4 & 26.5 \\
\hline Temperatura del suelo $\left({ }^{\circ} \mathrm{C}\right)$ & 24.7 & 23.9 & 26.3 & 24.7 & 22.7 & 23.2 & 22.5 & 23.1 & 22.6 \\
\hline Precipitación (mm) & 11.5 & 3.8 & 26.5 & 56.5 & 120.4 & 295 & 196 & 275 & 159 \\
\hline
\end{tabular}

Tabla 3

Análisis de similitud (ANOSIM) entre las épocas de muestreo en RCM, Atlántico, Colombia.

\begin{tabular}{lll}
\hline Comparaciones entre épocas & Estadístico $R$ & Nivel de significancia $(p)$ \\
\hline Seca-Primeras lluvias & 0.802 & 0.012 \\
Seca-Fuertes lluvias & -0.167 & 0.929 \\
Primeras lluvias-Fuertes lluvias & 0.293 & 0.006 \\
\hline
\end{tabular}

indica que el número efectivo de especies que son abundantes y muy abundantes presentan un patrón temporal.

El análisis de similitud (Anosim) demostró que existen diferencias significativas de la estructura de la comunidad de escarabajos fitófagos entre las épocas de muestreo $(R=0.27$, $p=0.002$ ). Las épocas más disímiles corresponden a las primeras lluvias con la época seca en la RCM (tabla 3). La especie que más contribuyó a la abundancia en las épocas de muestreo fue Liogenys quadridens con mayores porcentajes en el primer y último mes de su aparición en las muestras recolectadas (tabla 4). Seguidamente, se encuentra Phyllophaga pachypyga, con la mayor contribución en junio, debido a las bajas proporciones con que se presentan el resto de las especies recolectadas en el mes.

El gremio predominante fue rizofilófago, con el $45.5 \%$ de las especies, integrado por los taxones pertenecientes a la subfamilia Melolonthinae y algunas de Rutelinae (Geniatini). Los rizofilófagos alcanzan los valores máximos de riqueza y abundancia en abril y mayo, aunque se encuentran presentes en todos los meses de muestreos (figs. 3A y B). La especie P. didymus, categorizada como xilodepredador, apareció en los meses de mayor riqueza y abundancia, al igual que las especies saprocaulófagas, tales como T. fossor y S. aloeus. Los sapromelífagos (Cyclocephala spp.) y los rizofilófagos (Leucothyreus spp. y
Xenopelidnota anomala) parecen no presentar un patrón definido (fig. 3A). El análisis de correspondencia canónica (fig. 4; tabla 5) corrobora la influencia de los valores de precipitación sobre la aparición de L. quadridens durante los meses de muestreo. Phyllophaga pachypyga, Paranomala sp.1, T. fossor y $P$. didymus se ven influenciadas por los valores de temperatura ambiental y del suelo.

\section{Discusión}

El número de especies capturadas corresponde al $3.7 \%$ registradas en el territorio colombiano por Gasca-Álvarez y Amat-García (2010) y Restrepo-Giraldo et al. (2003). En el Caribe colombiano, los escarabajos fitófagos capturados representan el $39.3 \%$ de las descritas por Restrepo-Giraldo et al. (2003) y el 56\% de los registrados por García-Atencia y Martínez-Hernández (2015) en el departamento del Atlántico. Al tratarse de un estudio en ambientes silvestres, esta diversidad, considerada muy moderada, se ajusta de forma notable a los registros de las tierras agrícolas del Caribe, en las cuales se han registrado 2 tendencias: a) el Caribe húmedo (+/- $100 \mathrm{~m} \mathrm{snm})$ Montería, Córdoba (20 spp.), Tierra Alta, Córdoba, (21 spp.) y Caucasia, Antioquia (20 spp.) con un complejo Melolonthidae mejor estructurado y caracterizado por Pentodontini (Euetheola y Tomarus), Cyclocephalini (Cyclocephala y Dyscinetus) y Rutelinae-Anomalini (= Paranomala); algunos de ellos compartidos con regiones vecinas más húmedas, y b) el Caribe seco (13 especies en Majagual, Sucre, 10 en San Alberto César y 7 en Fonseca, Guajira) (Pardo-Locarno et al., 2012), región en la cual se asume que declina de forma notable la estructura del complejo de escarabajos edafícolas a una decena de especies asociadas a ambientes agrícolas muy intervenidos, como potreros, plantaciones de palma aceitera y otros monocultivos, con 
Tabla 4

Porcentaje de similitud (SIMPER) de especies de escarabajos fitófagos que caracterizan cada una de las épocas de muestreo en la RCM.

\begin{tabular}{llll}
\hline Especies/épocas & & Contribución (\%) \\
\cline { 2 - 4 } & Seca & Primeras lluvias & Fuertes lluvias \\
\hline Liogenys quadridens & 100 & 29.99 & 62.71 \\
Phyllophaga pachypyga & & 26.58 & 12.27 \\
Astaena sp. & & 8.11 & 7.91 \\
Phyllophaga menetriesi & & 5.6 & 15.75 \\
Paranomala sp.3 & & & 15.75 \\
Phyllophaga impressipyga & & 50.89 & 11.34 \\
Cyclocephala melanocephala & 100 & & \\
Xenopelidnota anomala & & & \\
Similitud promedio & & &
\end{tabular}
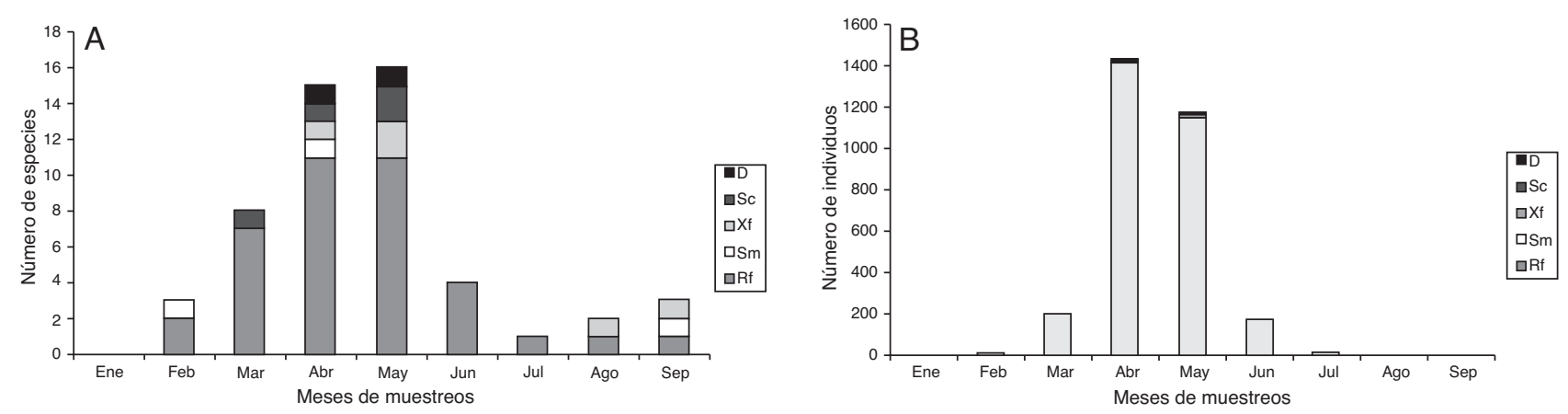

Figura 3. A, variación temporal de la riqueza y B, abundancia de los gremios de Scarabaeidae fitófagos en Reserva Campesina la Montaña. D: xilodepredador; Rf: rizofilófago; Sc: saprocaulófago; Sm: sapromelífago; Xf: xilofilófago.

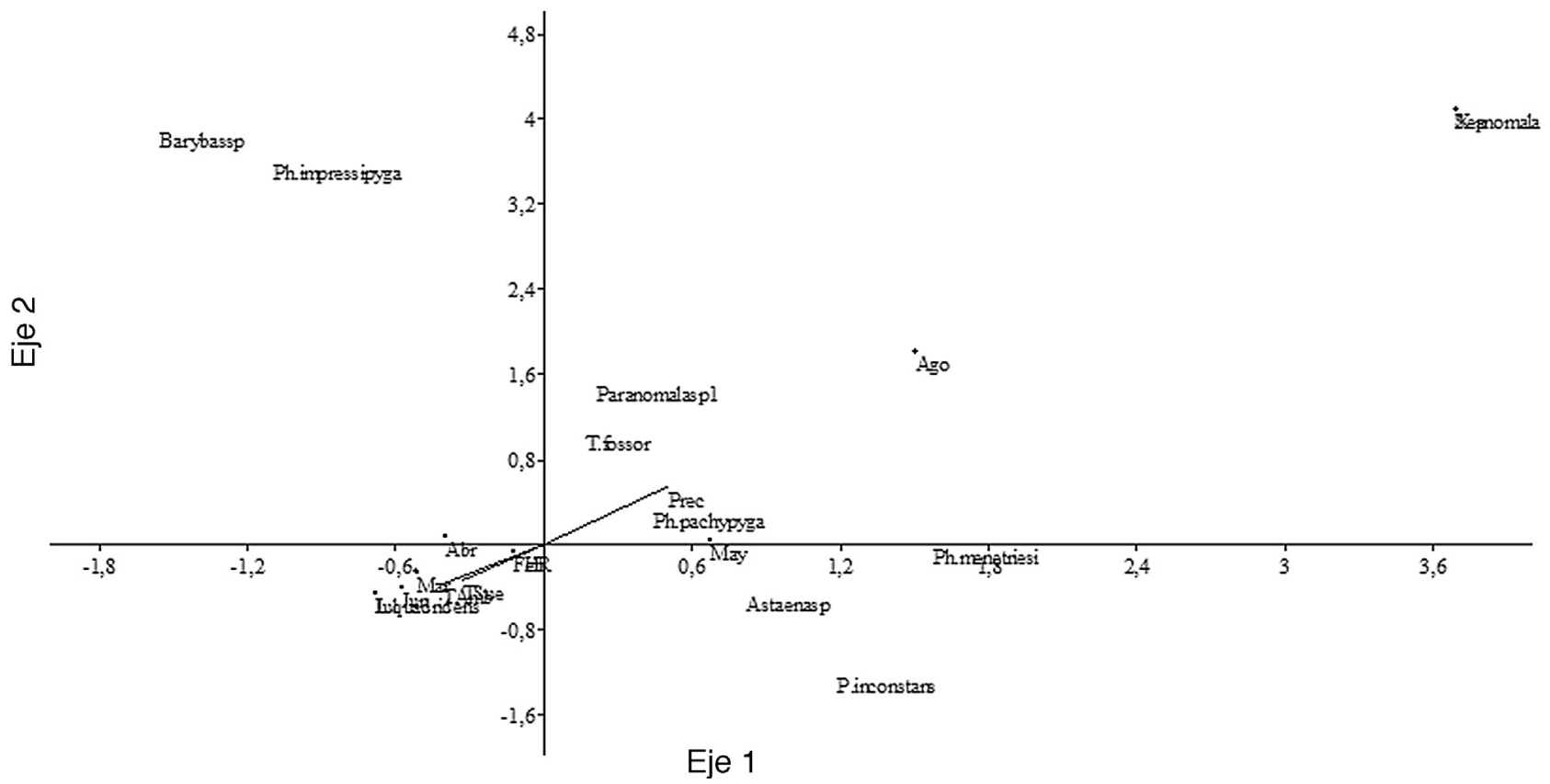

Figura 4. Análisis de correspondencia canónica entre variables ambientales y especies que aportaron más del 1\% de la abundancia total en la Reserva Campesina la Montaña, Atlántico, Colombia.

predominio poblacional de algunos grupos como Euetheola, Cyclocephala y Liogenys. En esta región, caracterizada por veranos intensos y largos, en los escarabajos edafícolas se observan periodos reproductivos marcadamente estacionales (Pardo-Locarno, Morón, Gaigl y Belloti, 2003).
$\mathrm{La}$ alta riqueza y abundancia de la subfamilia Melolonthinae se puede atribuir inicialmente, al menos a escala poblacional, al tipo de muestreo, el cual favoreció a este grupo y, además, a que es considerada la subfamilia más diversa en todo el mundo, con alrededor de 10,000 especies conocidas a escala mundial 
Tabla 5

Variación explicada por las variables ambientales, abundancia de las especies y por muestreo en cada uno de los ejes en el análisis de correspondencia canónica (ACC) en el fragmento de la RCM, Atlántico.

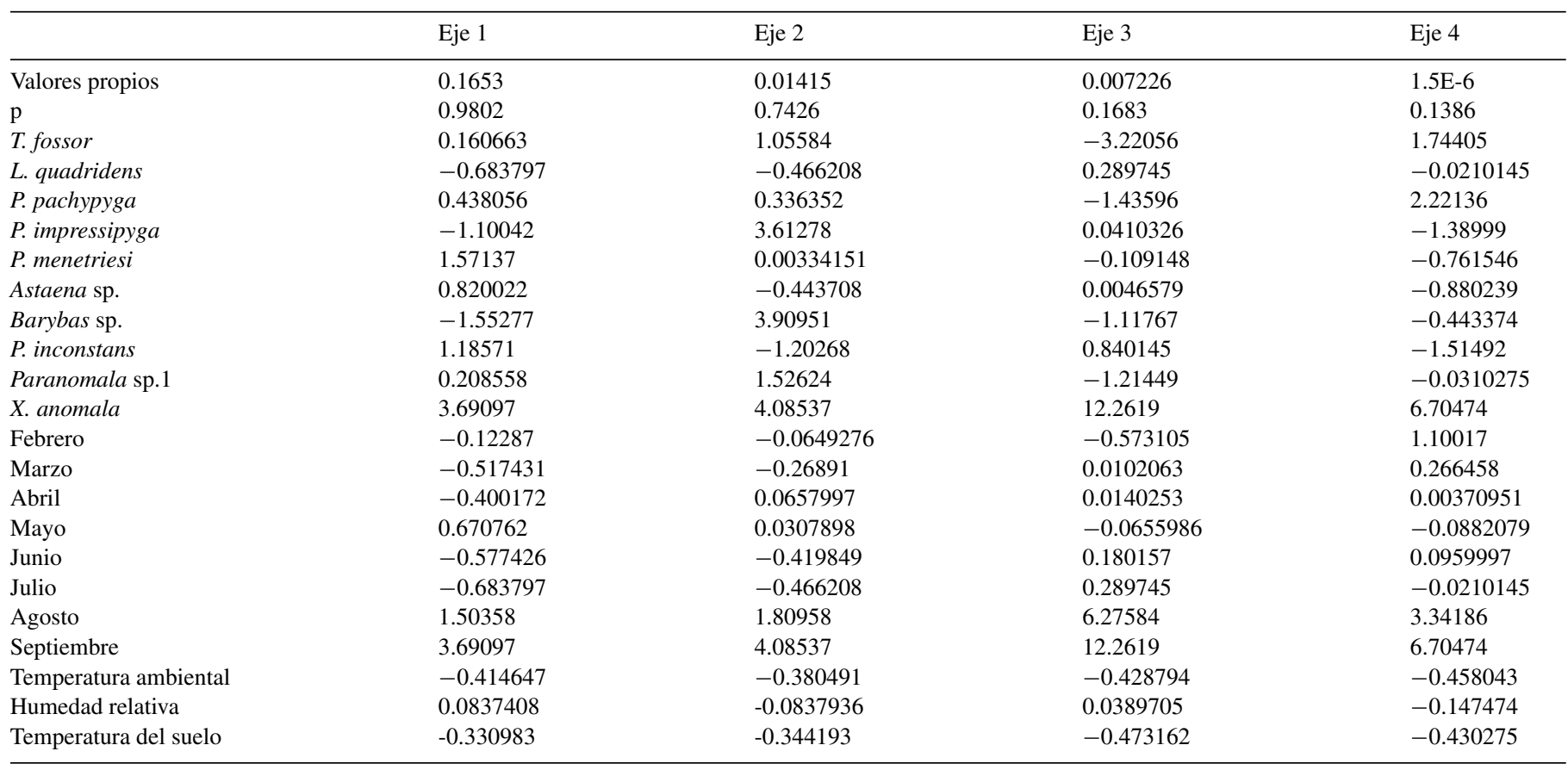

y con un amplio reconocimiento en la agricultura por sus larvas consumidoras de raíces, tallos, bulbos, tubérculos o materia orgánica (Morón, Ratcliffe y Deloya, 1997). Esta disponibilidad de recursos se puede encontrar en paisajes del departamento del Atlántico, en especial cuando en la matriz se encuentra un fragmento de bosque y cercas vivas que contribuyan a aumentar la diversidad de hábitats y proporcionan alimento para mantener una mayor abundancia de este grupo de escarabajos. Sin embargo, la degradación del hábitat que trae consigo su homogeneización pone en riesgo las poblaciones de especies con hábitos de alimentación y refugios restringidos, que podrían desaparecer con la extensión de las actividades antrópicas en la zona de estudio, fenómeno que se ha evidenciado con los escarabajos del bosque seco del alto Cauca (Pardo-Locarno, 2013). La baja riqueza de Dynastinae se atribuye a la falta de técnicas de muestreo específicas para capturar aquellas especies no fototrópicas que escasamente son recolectadas por trampas de luz. En la trayectoria de la curva de acumulación de especies, los singletons y doubletons varían poco durante los muestreos realizados, lo que demuestra que es necesario utilizar más técnicas de muestreo para disminuir la rareza de las muestras.

La alta abundancia de Melolonthinae se vio reflejada por $L$. quadridens y Phyllophaga menetriesi, que aportaron el 77.5\% a la abundancia total, lo que refleja una alta dominancia y la capacidad que tienen estas especies para adaptarse a lugares donde se presenta una degradación del hábitat. También puede deberse a la amplia disponibilidad de recursos alimenticios para estas especies en el fragmento, debido a que por ser fitófagas prefieren alimentarse del follaje, néctar y material vegetal en descomposición, elementos que abundan en una matriz compuesta por cultivos aledaños, lo que les garantiza el éxito reproductivo reflejado en sus altas abundancias. Además, la primera especie presenta una alta distribución en las tierras calientes de la Llanura del Caribe y se incluye como una importante plaga en el sorgo y el maíz (Pardo-Locarno, 2000; Pardo-Locarno et al., 2012), cultivos que son elementos del paisaje predominante en el área de estudio. Por otro lado, las diferencias en riqueza y abundancia por espectro de luz demuestran que las técnicas se comportaron como complementarias para capturar la mayor cantidad de especies en el área de estudio. Lo anterior corroboró lo descrito por García-López, Micó, Zumbado y Galante (2011), quienes, mediante pruebas de eficacia, sugirieron las trampas de luz como el mejor método de captura en estudios de biodiversidad en bosques tropicales. Durante mayo y abril se observó un incremento importante del número de individuos de las especies de Phyllophaga, que se caracterizan por emerger de las pupas en estos meses, por lo que son comúnmente conocidos como los "cucuyos de mayo". Esta es su época de reproducción, cuando aumentan su actividad, tal como lo ha descrito Morón (1994) en México y han registrado Buss (2006) y Martínez, Franz y Acosta (2009) en la Florida y Puerto Rico, respectivamente. Además, se nota el incremento del resto de individuos presentes en la época, cuando hay un aumento en la disponibilidad de refugio en el fragmento de bosque y se presentan las condiciones óptimas para la reproducción, como la disponibilidad de recursos alimenticios (Alcázar-Ruiz, Morón y Morón, 2003; Pardo-Locarno, 2013) y una distribución espacial más homogénea de las fuentes de alimento en el BST (Martínez, García et al., 2010). Alcázar et al. (2003) documentaron resultados similares en bosques subcaducifolios de México, que presentan la mayor riqueza y abundancia de los adultos de Phyllophaga en abril y 
mayo, y observaron una relación directa con la temporada de lluvias.

En marzo, el número de especies coincidió con el inicio de la época de primeras lluvias en la zona. En este muestreo se presentaron cambios fenológicos más evidentes en la vegetación, tales como aparición del follaje, lo que proporciona los primeros recursos utilizados por los adultos de la familia, quienes sincronizan sus ciclos de vida para garantizar su éxito reproductivo en la época de mayor disponibilidad de alimentos, tal como lo hacen otras especies y familias de coleópteros. Por el contrario, el resto de meses, es decir, los 3 últimos, se presentaron valores altos de precipitación y los valores de riqueza decrecieron, lo cual se debe a que la actividad de los escarabajos disminuye, aumenta su mortalidad y disminuye la efectividad de las trampas, tal como lo describieron Martínez et al. (2009) y Pinheiro, Diniz, Coelho y Bandeira (2002) en bosques de Brasil y Puerto Rico, respectivamente.

Las diferencias significativas entre las épocas demarcan una sincronización de los ciclos reproductivos de especies como $L$. quadridens, Phyllophaga spp. y Paranomala sp.1 en la RCM, debido a que dependen estrechamente de las lluvias en la zona. Esta variable explica en gran medida la estacionalidad de los individuos, ya que está relacionada con la productividad del bosque, del cual dependen los escarabajos fitófagos. Por su parte, C. melanocephala no presentó una marcada estacionalidad, lo que posiblemente indicaría que los recursos indispensables para su supervivencia pueden estar presentes todo el año. Algunas especies de este género se han encontrado en flores de Araceae, donde encuentran microhábitats propicios para su reproducción y alimentación (Gasca-Álvarez, 2013; Morón, 1997), así como en floraciones de plantas cultivadas y silvestres (Pardo-Locarno, 2013). Estos resultados son similares a los obtenidos por Neita et al. (2006) en el Chocó y Útima y Vallejo (2008) en agro-ecosistemas cafeteros (Colombia), donde C. melanocephala no mostró evidencias de estacionalidad, sino que se presentó en épocas con distintos valores de precipitación.

La mayor diversidad (serie de números de Hill) en la época de primeras lluvias para $\mathrm{N}_{1}$ demuestra que la frecuencia de las especies abundantes aumenta; éstas contribuyen de manera efectiva a la diversidad para esta época. Con este número de Hill se evalúa de foram eficaz la diversidad de especies ponderadas de acuerdo con su abundancia relativa; se observa que los valores del número efectivo de especies varían mucho entre las épocas de muestreos, lo que indica que las especies comunes se distribuyen de forma más heterogénea, al ser un fragmento de bosque que cambia con la llegada de las lluvias. Sin embargo, los valores para $\mathrm{N}_{2}$ pueden sugerir determinadas condiciones o ambientes de bosque seco normales con predominio de 1 o 2 especies, lo que demuestra que este índice toma valores bajos cuando las comunidades bióticas contienen pocas especies que son abundantes y muchas que son escasas (Marcelo-Peña, Rodríguez, Zevallos-Pollito, Bulnes-Soriano y Pérez-Ojeda Del Arco, 2007), y cuando las muestras son heterogéneas (Magurran, 2004).
En relación con los gremios tróficos, los rizofilófagos estuvieron presentes en todos los meses de muestreos, con valores de riqueza y abundancia elevados, sobre todo en abril y mayo, meses en los cuales la disponibilidad de recursos que utilizan como raíces jóvenes y material vegetal en descomposición son los más comunes en la RCM. Durante ese periodo, se da la proliferación de raíces jóvenes, renovación y brotes de hojas que son preferidas por los herbívoros, debido a que su contenido de agua y nitrógeno suele ser mayor que el de una hoja madura, lo que aumenta la tasa de herbivoría (Martínez, Cañas et al., 2010).

Resultados similares se obtuvieron en un fragmento aledaño, en el que los fitófagos, representados en su gran mayoría por la familia Scarabaeidae "pleurosticti", alcanzaron los mayores valores para los mismos meses (Martínez, García et al., 2010). Según Alcázar et al. (2003), los roles dominantes que no desaparecen en el tiempo tienen una mayor importancia para el constante funcionamiento del ecosistema, aunque la desaparición de roles no tan comunes podría afectar los procesos en una escala temporal a largo plazo. Por esto, los gremios más susceptibles a las alteraciones causadas en la RCM son aquellos cuyas representaciones en este estudio obtuvieron mínimos valores de abundancia y riqueza, como es el caso de depredadores, sapromelífagos y saprocaulófagos.

El gremio de depredadores estuvo representado por $P$. didymus presente en abril y mayo, que se considera como un gremio funcional raro en esta familia, lo que lo hace más vulnerable a las extinciones locales. Sin embargo, la técnica de captura pudo influir en la recolección de pocos individuos de esta especie, por lo que sería pertinente comprobar la rareza o no de esta especie. La alta riqueza de gremios tróficos presentada en estos meses se debe al inicio de la temporada de lluvias, la acumulación del material vegetal y su descomposición, lo que propicia la existencia de recursos alimenticios para los diferentes gremios de Scarabaeidae.

En los fragmentos de BST, la variación climática es muy grande entre la temporada seca y la de lluvias, lo que incide en los valores de humedad y temperatura, que son importantes en la regulación de las comunidades de insectos en estos ecosistemas tropicales (Pardo-Locarno, 2013; Prince-Chacón, Vargas-Zapata, Salazar y Martínez, 2011); esto puede explicar la correlación entre estas variables con algunas de las especies que aparecieron en este periodo.

\section{Agradecimientos}

A la Universidad del Atlántico por facilitarnos sus instalaciones y algunas herramientas indispensables para la culminación de esta investigación. Al semillero de Investigación de Artrópodos e Insectos NEOPTERA del grupo de Investigación Biodiversidad del Caribe colombiano por sus aportes y colaboración. A la comunidad campesina de la Montaña, especialmente a Santiago y Rafael Conrado, por su atención durante los meses de muestreo. A María Angélica Vargas, por facilitar el mapa del área de estudio. 
Anexo. Especies de escarabajos y su abundancia mensual en la RCM

\begin{tabular}{|c|c|c|c|c|c|c|c|c|c|c|}
\hline \multirow[t]{2}{*}{ Especies } & \multicolumn{9}{|c|}{ Meses de muestreo } & \multirow[t]{2}{*}{ Total } \\
\hline & Enero & Febrero & Marzo & Abril & Mayo & Junio & Julio & Agosto & Septiembre & \\
\hline \multicolumn{11}{|l|}{ Dynastinae $(S=5, N=18)$} \\
\hline Cyclocephala melanocephala (Fabricius, 1775) & 0 & 2 & 0 & 0 & 0 & 0 & 1 & 0 & 1 & 4 \\
\hline C. ovulum Bates, 1888 & 0 & 0 & 0 & 2 & 0 & 0 & 0 & 0 & 0 & 2 \\
\hline \multicolumn{11}{|l|}{ Pentodontini } \\
\hline Tomarus fossor (Latreille, 1833) & 0 & 0 & 1 & 4 & 3 & 0 & 0 & 0 & 0 & 8 \\
\hline \multicolumn{11}{|l|}{ Phileurini } \\
\hline Phileurus didymus Linnaeus, 1758 & 0 & 0 & 0 & 1 & 1 & 0 & 0 & 0 & 0 & 2 \\
\hline \multicolumn{11}{|l|}{ Melolonthinae $(S=6, N=2,907)$} \\
\hline \multicolumn{11}{|l|}{ Melolonthini } \\
\hline Phyllophaga impressipyga (Frey, 1975) & 0 & 0 & 1 & 100 & 26 & 0 & 0 & 0 & 0 & 127 \\
\hline P. (P.) menetriesi (Blanchard, 1851) & 0 & 0 & 0 & 116 & 549 & 0 & 0 & 0 & 0 & 665 \\
\hline Barybas sp.1 & 0 & 0 & 2 & 33 & 2 & 0 & 0 & 0 & 0 & 37 \\
\hline Liogenys quadridens (Fabricius, 1758) & 0 & 6 & 168 & 986 & 329 & 161 & 16 & 1 & 0 & 1,667 \\
\hline \multicolumn{11}{|l|}{ Rutelinae $(S=7, N=82)$} \\
\hline \multicolumn{11}{|l|}{ Anomalini } \\
\hline Paranomala inconstans (Burmeister, 1844) & 0 & 0 & 0 & 4 & 15 & 1 & 0 & 0 & 0 & 20 \\
\hline Paranomala sp.1 & 0 & 0 & 2 & 20 & 19 & 0 & 0 & 0 & 0 & 41 \\
\hline Paranomala sp.2 & 0 & 0 & 0 & 0 & 4 & 0 & 0 & 0 & 1 & 5 \\
\hline Paranomala sp. 3 & 0 & 0 & 0 & 0 & 1 & 0 & 0 & 0 & 0 & 1 \\
\hline \multicolumn{11}{|l|}{ Geniatini } \\
\hline Leucothyreus sp.1 & 0 & 0 & 0 & 1 & 0 & 0 & 0 & 0 & 0 & 1 \\
\hline Leucothyreus sp. 2 & 0 & 0 & 0 & 0 & 1 & 0 & 0 & 0 & 0 & 1 \\
\hline \multicolumn{11}{|l|}{ Rutelini } \\
\hline
\end{tabular}

\section{Referencias}

Alcázar-Ruiz, J., Morón, A. y Morón, M. A. (2003). Fauna de Coleoptera Melolonthidae de Villa Las Rosas, Chiapas, México. Acta Zoológica Mexicana, $88,59-86$.

Buss, E. (2006). Flight activity and relative abundance of phytophagous scarabs (Coleoptera: Scarabaeoidea) from two locations in Florida. Florida Entomologist, 89, 32-40.

Cano, E. B. y Schuster, J. C. (2009). Beetles as indicators for forest conservation in Central America International Commission on Tropical Biology and Natural Resources. Encyclopedia of Life Support Systems (EOLSS),. Recuperado de http://www.eolss.net/ebooks/Sample\%20Chapters/C20/E6142-TPE-04.pdf. Consultado 12 de agosto de 2015

Castellanos, M. L., Pardo-Locarno, L. C., Carabalí, A. y Doria, C. (2011). Algunas características de macrofauna del suelo en la Serranía de la Macuira, Guajira, Colombia. Revista Agricultura Tropical, 34, 98-106.

Clarke, K. R. y Warwick, R. M. (2001). Change in marine communities: an approach to statistical analysis and interpretation (Segunda edición). Plymouth: PRIMER-E.

Colwell, R.K. (2013). Estimates: statistical estimation of species richness and shared species from samples. Ver. 9.1. User's guide and application. Recuperado de http://purl.oclc.org/estimates

Deloya, C., Parra-Rabla, V. y Delfín-González, H. (2007). Fauna de los coleópteros Scarabaeidae Laparosticti y Trogidae (Coleptera: Scarabaeoidaea) asociados al bosque mesófilo de montaña, cafetales bajo sombra y comunidades derivadas en el centro de Veracruz, México. Neotropical Entomology, 36, 5-21.

Delgado, L., Pérez, A. y Blackaller, J. (2000). Claves para determinar a los taxones genéricos y supragenéricos de Scarabaeoidea Latreille, 1802 de México. Folia Entomológica Mexicana, 110, 33-87.

Endrödi, S. (1985). The Dynastinae of the World. Dordrecht/Boston/Lancaster: Dr. W. Junk Publishers.

Escobar, F. (1997). Estudio de la comunidad de coleópteros (Scarabaeidae) en un remanente del bosque seco del Norte del Tolima, Colombia. Caldasia, $19,419-430$

García-López, A., Micó, E., Zumbado, M. A. y Galante, E. (2011). Sampling scarab beetles in tropical forests: the effect of light source and night sampling periods. Journal of Insect Science, 11, 95.

Gasca-Álvarez, H. J. (2013). New records of Cyclocephala Dejean (Coleoptera: Scarabaeidae: Dynastinae) associated whit Caladium bicolor (Aiton) Vent. (Araceae). The Coleopterists Boulletin, 6, 416-418.

Gasca-Álvarez, H. J. y Amat-García, G. (2010). Synopsis and key to the genera of Dynastinae (Coleoptera, Scarabaeoidea, Scarabaeidae) of Colombia. En B. Ratcliffe y F. T. Krell (Eds.), Current advances in Scarabaeoidea research. ZooKeys, 34, 153-192.

García-Atencia, S. y Martínez-Hernández, N. J. (2015). Escarabajos fitófagos (Coleoptera: Scarabaeidae) del departamento del Atlántico. Acta Zoológica Mexicana (nueva serie), 31, 89-96.

Hernández, J. (1992). Caracterización geográfica de Colombia. En G. Halffter (Ed.), La diversidad biológica de Iberoamérica I (pp. 45-54). México, 
D.F.: CYTED-B Programa Iberoamericano de Ciencia y Tecnología para el Desarrollo. Instituto de Ecología, A.C.

Holdridge, L. R., Grenke, W. C., Hatheway, W. H., Liang, T. y Tosi, J. A. (1971). Forest environments in tropical life zones, a pilot study. Oxford: Pergamon Press.

IAVH (Instituto Alexander Von Humboldt). (1998). El bosque seco tropical (bs-T) en Colombia. Villa de Leyva: Grupo de Exploraciones y Monitoreo Ambiental GEMA.

Lassau, S. A., Hochuli, D. F., Cassis, G. y Reid, C. A. M. (2005). Effects of habitat complexity on forest beetle diversity: do functional groups respond consistently? Diversity and Distributions, 11, 73-82.

Londoño, M. (1999). Complejo de chisas en Colombia y perspectivas para su manejo. En: Memorias del XXVI Congreso de la Sociedad colombiana de Entomología (SOCOLEN), Bogotá, julio 1999.

Magurran, A. E. (2004). Measuring biological diversity. Oxford: Blackwell Science.

Marcelo-Peña, J. L., Rodríguez, C. R., Zevallos-Pollito, P., Bulnes-Soriano, F. y Pérez-Ojeda Del Arco, A. (2007). Diversidad, composición florística y endemismos en los bosques estacionalmente secos alterados del distrito de Jaén, Perú. Ecología Aplicada, 6, 9-22.

Martínez, N., Cañas, L., Rangel, J., Barraza, J., Montes, J. y Blanco, O. (2010). Coleópteros coprófagos (Scarabaeidae: Scarabaeinae) en un fragmento de bosque seco tropical en el departamento del Atlántico, Colombia. Boletín del Museo de Entomología, Universidad del Valle, 11, 21-30.

Martínez, N., García, S., Sanjuán, S., Gutiérrez, M. J. y Contreras, C. (2010). Composición y estructura de la fauna de escarabajos (Insecta: Coleoptera) atraídos por trampas de luz en La Reserva Ecológica de Luriza, Atlántico, Colombia. Boletín de la Sociedad Entomológica Aragonesa, 47, 373-381.

Martínez, N. J., Franz, N. y Acosta, J. (2009). Structure of the beetle fauna (Insecta: Coleoptera) in forest remmants localized on the campus of the University of Puerto Rico at Mayagüez. Entomotropica, 93, 83-100.

Miles, L., Newton, A. C., Defries, R. S., Ravilious, C., May, I., Blyth, S., et al. (2006). A global overview of the conservation status of tropical dry forests. Journal of Biogeography, 33, 491-505.

Morón, M. A. (1985). Los insectos degradadores, un factor poco estudiado en los bosques de México. Folia Entomológica Mexicana, 65, 131-137.

Morón, M. A. (1994). Fauna de Coleoptera Lamellicornia en las montañas del noreste de Hidalgo, México. Acta Zoológica Mexicana (nueva serie), 63, $7-59$.

Morón, M. A. (1997). Notas sobre Cyclocephala Latreille (Coleoptera: Melolonthidae: Dynastinae) asociadas con Xanthosoma Schott (Araceae) en Chiapas, México. Giornale Italiano di Entomologia, 8, 399-407.

Morón, M. A. y Deloya, C. (1991). Los Coleoptera Lamellicornia de la Reserva de la Biosfera "La Michilía", Durango, México. Folia Entomológica Mexicana, 81, 209-283.

Morón, M. A., Ratcliffe, B. y Deloya, C. (1997). Atlas de los escarabajos de México: Coleoptera Lamellicornia. Vol. I. Familia Melolonthidae. Xalapa: Sociedad Mexicana de Entomología, A.C.

Neita, J. C., Orozco, J. y Ratcliffe, B. (2006). Escarabajos (Scarabaeidae: Pleurosticti) de la selva del bosque pluvial tropical-BP-T, Chocó, Colombia. Acta Zoológica Mexicana, 22, 1-32.

Panza, E., Torres, M., De la Roda, P., Berdugo, I., Torres, A., De la Rosa, P., et al. (2001). Esquema de ordenamiento territorial municipio de Juan de Acosta. Juan de Acosta: Departamento del Atlántico: Alcaldía municipal de Juan de Acosta 2001-2009.

Pardo-Locarno, L. C. (2000). Avances en el estudio de chisas rizófagas (Coleoptera-Melolonthidae) en Colombia, observaciones sobre los complejos regionales y nuevos patrones morfológicos de larvas. Santiago de
Cali: III Curso Nacional sobre Taxonomía de Escarabajos de Importancia Económica en Colombia.

Pardo-Locarno, L. C. (2013). Escarabajos (Coleoptera: Melolonthidae) del plan aluvial de Río Cauca, Colombia I. Ensamblaje, fichas bioecológicas, extinciones locales y clave para adultos. Dugesiana, 20, 1-15.

Pardo-Locarno, L. C., González, J. C., Pérez, C. R., Yepes, F. y Fernández, C. (2012). Escarabajos de importancia agrícola (Coleoptera: Melolonthidae) en la región Caribe colombiana: registros y propuestas de manejo. Boletín del Museo Entomológico Francisco Luis Gallejo, 4, 7-23.

Pardo-Locarno, L. C., Montoya, J. y Schoonhoven, A. (2003). Comparación de la abundancia de chisas rizófagas (Coleoptera: Melolonthidae) en agroecosistemas de Caldono, Cauca, Colombia. En G. A. Aragón, M. A. Morón y A. J. Marín (Eds.), Estudios sobre coleópteros del suelo en América (pp. 65-77). Puebla: Publicación especial de la Benemérita Universidad Autónoma de Puebla.

Pardo-Locarno, L. C., Montoya, J., Schoonhoven, A. y Morón, M. A. (2003). Composición y riqueza del complejo Melolonthidae (Coleoptera) en cuatro agroecosistemas del Cauca, Colombia. En G. A. Aragón, M. A. Morón y A. J. Marín (Eds.), Estudios sobre coleópteros del suelo en América (pp. 29-43). Puebla: Publicación especial de la Benemérita Universidad Autónoma de Puebla.

Pardo-Locarno, L. C., Morón, M. A., Gaigl, A. y Belloti, A. (2003). Los complejos regionales de Melolonthidae (Coleoptera) rizófagos en Colombia. En G. A. Aragón, M. A. Morón y A. J. Marín (Eds.), Estudios sobre coleópteros del suelo en América. (pp. 45-63). Puebla: Publicación especial de la Benemérita Universidad Autónoma de Puebla.

Pinheiro, F., Diniz, I. R., Coelho, D. y Bandeira, M. P. S. (2002). Seasonal pattern of insect abundance in the Brazilian Cerrado. Austral Ecology, 27, 132-136.

Portillo-Quintero, C. A. y Sánchez-Azofeifa, Y. G. A. (2010). Extent and conservation of tropical dry forests in the Americas. Biological Conservation, 143, 144-155.

Prince-Chacón, S., Vargas-Zapata, M. A., Salazar, J. y Martínez, N. J. (2011). Mariposas Papilionoidea y Hesperioidea (Insecta: Lepidoptera) en dos fragmentos de bosque seco tropical en Corrales de San Luis, Atlántico, Colombia. Boletín de la Sociedad Entomológica Aragonesa, 48, 243-252.

Ramírez-Ponce, A. y Morón, M. A. (2009). Relaciones filogenéticas del género Anomala (Coleoptera: Melolonthidae: Rutelinae). Revista Mexicana de Biodiversidad, 80, 357-394.

Restrepo-Giraldo, H., Morón, M. A., Vallejo, F., Pardo-Locarno, L. C. y LópezÁvila, A. (2003). Catálogo de Coleoptera Melolonthidae (Scarabaeidae Pleurosticti) de Colombia. Folia Entomológica Mexicana, 42, 239-263.

Sanabria-García, R., Gasca-Álvarez, H. J. y Amat-García, G. (2012). Sinopsis de la Tribu Oryctini (Coleoptera: Scarabaeidae: Dynastinae) de Colombia. Insecta Mundi, 0276, 1-64.

Schëu, S. (2002). The soil food web: structure and perspectives. European Journal of Soil Biology, 38, 11-20.

Suárez, M. A. y Amat, G. (2007). Lista de especie de los escarabajos fruteros (Melolonthidae: Cetoniinae) de Colombia. Biota Colombiana, 8, 69-76.

Útima, O. y Vallejo, L. F. (2008). Escarabajos Melolonthidae (ScarabaeidaePleurosticti) de la montaña cafetera, departamento de Risaralda, Colombia. Agronómica, 16, 31-44.

Vallejo, L. F. y Wolff, M. (2013). The genus Phyllophaga Harris (Coleoptera: Scarabaeidae: Melolonthinae) in the Colombian Andean Mountain. Zootaxa, 3722, 101-142.

Yepes, F., Pardo, L., Pérez, C. y Quiroz, J. (2000). Contribución al reconocimiento de especies de escarabajos (Coleoptera: Scarabaeoidea) en el departamento de Antioquia. Congreso de la Sociedad Colombiana de Entomología. Universidad Nacional, Sede Medellín, 351-376. 